\title{
MAPPING THE UPDATING PROCESS: COMMON AND SPECIFIC BRAIN ACTIVATIONS ACROSS DIFFERENT VERSIONS OF THE RUNNING SPAN TASK
}

\author{
Fabienne Collette ${ }^{1,2}$, Martial Van der Linden ${ }^{1,4}$, Steven Laureys ${ }^{2}$, Flavia Arigoni ${ }^{1}$, Guy Delfiore ${ }^{2}$, Christian \\ Degueldre $^{2}$, André Luxen ${ }^{2}$ and Eric Salmon ${ }^{2,3}$ \\ ${ }^{I}$ Neuropsychology Unit, University of Liège, Liège, Belgium; ${ }^{2}$ Cyclotron Research Centre, University of Liège, \\ Liège, Belgium; ${ }^{3}$ Department of Neurology, University of Liège, Liège, Belgium; ${ }^{4}$ Cognitive Psychopathology \\ Unit, University of Geneva, Geneva, Switzerland
}

\begin{abstract}
Neuroimaging studies exploring the neural substrates of executive functioning have only rarely investigated whether the non-executive characteristics of the experimental executive tasks could contribute to the observed brain activations. The aim of this study was to determine cerebral activity in three different tasks involving the updating executive function. The experimental updating tasks required subjects to process strings of items (respectively letters, words, and sounds) of unknown lengths, and then to recall or identify a specific number of presented items. Conjunction and functional connectivity analyses demonstrated that the cerebral areas activated by all three experimental tasks are the left frontopolar cortex, bilateral dorsolateral prefrontal and premotor cortex, bilateral intraparietal sulcus, right inferior parietal lobule and cerebellum. Some regions of this network appear to be more specific to each updating task. These results clearly indicate that the neural substrates underlying a specific executive process (in this case, updating) are modulated by the exact requirements of the task (such as the material to process or the kind of response) and the specific cognitive processes associated with updating.
\end{abstract}

Key words: executive functions, updating, functional imaging, frontal, parietal

\section{INTRODUCTION}

Updating of working memory representations is frequently postulated to be an important executive function (e.g., Miyake et al., 2000; Shimamura, 2000). This function is often defined as a modification of the content of working memory by deleting no longer relevant information and incorporating more relevant data. This ability to update the content of working memory is required in many everyday activities and job environments, especially those involving multitasking such as cooking a multi-dish meal or controlling air traffic (Bjork, 1978). A series of data indicate that the updating function is clearly dissociable from other executive processes. Using latent variable analysis, Miyake et al. (2000) showed that the updating function could be considered as separable from shifting between mental sets or tasks and inhibition of prepotent responses, although there are some commonalities between these three processes. Fisk and Sharp (2004) explored the effect of age on executive functioning and obtained a factor structure that was globally consistent with that found by Miyake et al. (2000), and in particular with the identification of an updating factor that is relatively independent of three other executive factors (inhibition, shifting, and access to long-term memory). More recently, a dissociation between intact updating function and impaired manipulation of information in working memory (i.e., transforming the content of the information to be recalled) was observed in persons with Parkinson's disease (Gilbert et al., 2005), while a reverse dissociation was obtained in two different samples of healthy elderly (Belleville et al., 1998; Van der Linden et al., 1999b). Information concerning the status of the updating function as a relatively independent executive process has also been obtained by exploring its neural substrates. We recently conducted a positron emission tomography (PET) study to examine the cerebral areas associated with updating, shifting and inhibition using conjunction and interaction analyses (Collette et al., 2005). A global conjunction analysis demonstrated foci of activation common to all executive tasks, especially in the right intraparietal sulcus and the left superior parietal gyrus, supporting the hypothesis that the various executive processes share some common features. Interaction analyses also revealed that specific cerebral areas are associated with each executive process, and more specifically that the left frontopolar gyrus (BA 10) is associated with updating but not with the two other executive functions. 
More generally, a wide range of neuroimaging studies have explored the neural substrates of updating, using the running memory task and the n-back task. The running memory task (initially used by Pollack et al., 1959) requires participants to attend to a series of individual items (e.g., consonants) and retain the n most recent presented, for subsequent serial recall. Because the onset of the recall cue is unpredictable, subjects have to update their memory set with the presentation of each new item. Thus, this task requires a progressive shift of attention from internally represented information (the items maintained in working memory) to externally presented information. Morris and Jones (1990) showed that the running memory task requires two independent working memory mechanisms: the phonological loop, which is involved in the serial recall component of the task, and the central executive, which is involved in the updating process. In the n-back task, items (letters, spatial positions, or patterns) are sequentially presented and participants have to evaluate whether each item is similar to the one presented $\mathrm{n}$ items previously. Thus, the $\mathrm{n}$-back task is similar to the running span task, with the additional requirement that subjects must respond to each new item by evaluating it against the "oldest" item in the memory set.

The first neuroimaging studies to explore the neural substrates of the updating process used the n-back task. In a review of these studies (Collette and Van der Linden, 2002), we noticed that performance on that task was associated with cerebral activity not only in the prefrontal dorsolateral cortex (BA 9/46), inferior frontal cortex (BA 44), premotor cortex and anterior cingulate, but also in posterior cerebral areas, such as the superior and posterior parietal cortex (BA 40/7) (see, for example, Braver et al., 1997; Cohen et al., 1994, 1997; Jonides et al, 1997; Schumacher et al., 1996; Smith et al., 1996). However, when rehearsal processes were controlled, activity in the language areas (BA 44 and premotor cortex) disappeared (Awh et al., 1996). Moreover, the letter version of this task was associated with left (or bilateral) activations, whereas spatial or object versions tended to activate right-hemisphere regions (Owen et al., 1998; Smith et al, 1996). One limitation on these studies concerns the control tasks used. Indeed, memory load was equated for the control and experimental (updating) conditions by requiring participants to detect, in the control task, a number of target items similar to that which had to be maintained in working memory. However, a difference between the two conditions is that the n-back task requires one to update throughout the task the letters to be maintained in working memory while, in the control condition, the same items were maintained for the whole task. Thus, it could be argued that the control task became more automatic or that participants used long-term memory to perform it, while the experimental task required a continuous activation of the phonological loop. Consequently, some increases in activity observed during the n-back task might not be due to the updating process per se but rather to greater involvement of the phonological loop [as already observed by Awh et al. (1996) concerning the articulatory rehearsal process].

In order to more accurately dissociate the respective contributions of the phonological loop and the updating function and to allow a correct matching of the memory load in the control and experimental tasks, we conducted a PET study to compare changes in cerebral metabolism during performance on a phonological short-term memory task and the running memory task (Salmon et al., 1996). In the phonological task, participants were instructed to rehearse serially series of six consonants so that they could detect whether a target consonant, presented after a delay, was present in the list. In the updating condition, lists of 8, 9 and 10 consonants were presented and participants were not informed of the length of the lists. They were asked to rehearse silently and to remember serially only the six last items in order to decide whether a target consonant, presented after a delay, was present in the six last consonants for this particular list. When working memory updating was compared to phonological short-term memory, increases of activity occurred in the right mid-dorsal prefrontal cortex (BA 9), the left middle frontal regions (BA 46 and possibly BA 10) and the right frontal pole (BA 10). Increased regional cerebral blood flow (rCBF) was also found in a broad area of the right inferior parietal and angular gyri (BA 40/39), and in the left supramarginal gyrus (BA 40). However, a problem with this study was that subjects used a combination of phonological and visuospatial strategies to perform the running span task. As a result, some of the observed activation (e.g., parietal and occipital activation) might be related to the generation and short-term storage of visuospatial images rather than to central executive functioning. Moreover, the memory load of six items may have induced executive processes (for example, by grouping items into higher-level units) also in the control storage tasks as well. Consequently, we carried out a second PET experiment (Van der Linden et al., 1999a) to re-examine the brain regions involved in working memory updating by using a serial recall procedure instead of a recognition procedure and by using a sub-span (four-item) memory load, which is presumably less dependent upon the central executive of working memory. When working memory updating was compared to phonological short-term memory, the most significant increase in activity occurred in the left frontopolar cortex (BA 10). Activation spread to the left middle frontal cortex (BA 46) and was also observed in the right frontopolar cortex. Using a similar task with fMRI, Postle et al. (2001) argued that the discarding and repositioning operations assumed to be required for an updating task are not fundamentally different from the encoding- and maintenance-related processes that are engaged by all working memory tasks, since a similar network of cerebral regions (prefrontal cortex, superior frontal areas, anterior cingulate cortex, posterior parietal 
cortex, inferior temporal cortex, and occipital cortex) was found for updating and non-updating items. Finally, Cornette et al. (2001) showed that when the running memory task was performed with visual material, the updating process was associated mainly with increased activity in the superior frontal sulcus region (BA 6/8) but not in the frontopolar cortex.

Another problem with these neuroimaging studies is the use of task-specific analyses (or a subtraction design), where the specificity of active versus baseline differences for the cognitive function under study is often questionable (Friston et al., 1996; Sidtis et al., 1999). Indeed, a running memory task that mainly depends on the updating function probably also involves other executive processes to some extent, such as inhibition (or resistance to interference; see De Beni and Palladino, 2004) and shifting. This multi-compound aspect leads to major difficulties in finding an experimental task that enables one to isolate the specific process of updating, and developing a control task that suppresses the influence of both 'out-of-interest' executive processes and nonexecutive processes simultaneously.

\section{OBJECTIVES}

Since previous studies showed great variability in the neural substrates associated with updating, which could be related to methodological limitations listed above, the aim of the present study was to explore the unity and diversity of the neural substrate associated with this process. More specifically, we were interested in determining whether certain cerebral areas are common to several tasks requiring updating, whatever the idiosyncratic characteristics of the tasks administered (such as the kind of stimuli to be processed or the response modality). We capitalised on a cognitive study by Miyake et al. (2000) that used latent variable analysis to determine to what extent different executive functions can be considered to be unitary (in the sense that they are a reflection of the same underlying mechanism or ability) or nonunitary. These authors used a set of nine executive tasks to explore the separability of three functions often described as executive: updating, shifting and inhibition. The results indicated that these three functions were clearly separable at a cognitive level, although they did share some features in common. In a previous study (Collette et al, 2005), the tasks used by Miyake et al. (2000) were adapted to the PET methodology to determine the cerebral areas associated with these three executive processes. In the present study, we re-examined these neuroimaging data by focusing on the tasks associated with the updating factor. A conjunction analysis was used to isolate common cerebral areas activated by all three updating tasks.

Moreover, each updating task was compared to its matched control task to highlight the brain regions specifically associated with tasks requiring slightly different updating processes. The simultaneous use of conjunction and subtraction statistical designs should allow for a better characterisation of the cerebral areas that are both common to different tasks considered to reflect the same cognitive process, namely updating, and specific to each one. In addition to exploring the commonality and specificity of cerebral activity linked to various updating tasks, we were also interested in determining the functional connectivity between the network of cerebral areas associated with updating. Functional connectivity refers to correlations between remote neurophysiological events. Such an analysis had not yet been performed with updating tasks and the existence of covariance between specific cerebral areas should allow us to better understand the functional role of these areas in the context of updating. In our previous study on executive functioning (Collette et al., 2005), we did not tackle the question of the influence of the idiosyncratic characteristics of the tasks on the running of executive processes (in this case, updating), or the exploration of functional connectivity. So, the data obtained in the present study should improve our understanding of the neural substrates of the updating process.

\section{MATERIAL AND METHODS}

\section{Subjects}

Twelve right-handed European volunteers ( 6 males and 6 females, age range from 19 to 25 years) gave their written informed consent to take part in this study, which was approved by the University of Liège Ethics Committee. None had any past medical history or used any medication.

\section{Cognitive Tasks}

The experimental design comprised three conditions, each one consisting of an experimental updating task and a matched control task. The tasks used were adapted from the study by Miyake et al. (2000). The experimental updating tasks required subjects to process strings of items of unknown lengths, and then to recall or identify a specific number of the most recently presented items. Control tasks only required the temporary storage of items, 
without any need to update the presented information. The memory load was similar for the control and experimental task in each condition. In the first condition, subjects had to store and update lists of consonants. In the second condition, words were presented that had to be semantically processed, and in the third condition, subjects had to process sounds with different pitches.

Fig. 1 - Schematic illustration of cognitive components involved in consonants updating: (a) control task, (b) experimental updating task.

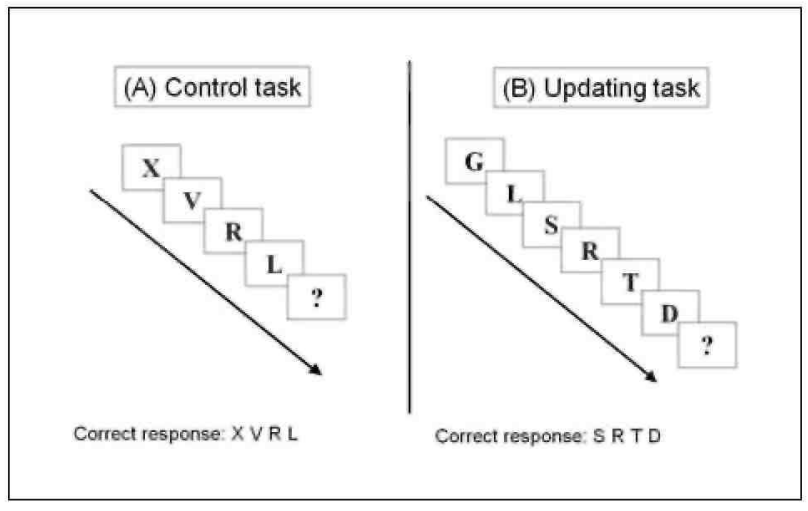

Fig. 2 - Schematic illustration of cognitive components involved in words updating: (a) control task, (b) experimental updating task.

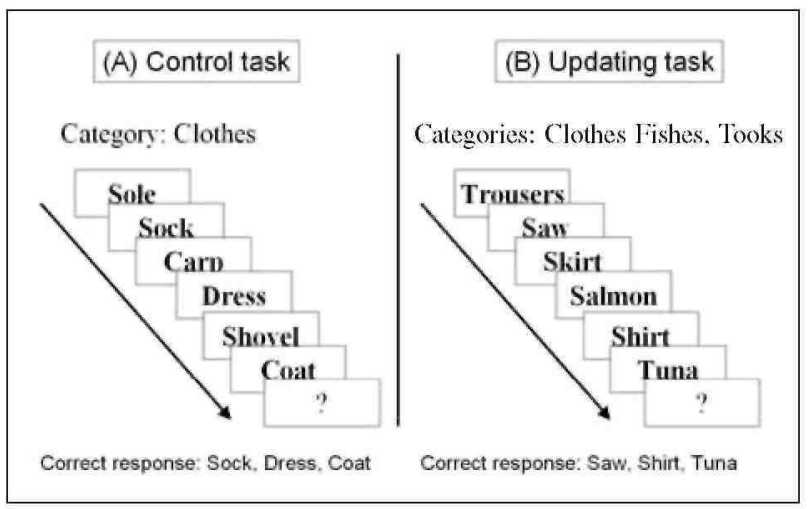

\section{Consonant Condition}

The material consisted of the 19 French monosyllabic consonants. These consonants were presented one at a time on a visual display (120-point Arial Narrow font) and subjects responded aloud.

In the control working-memory task, randomised sequences of four consonants were displayed on the computer screen at a rate of one every two seconds. Subjects were instructed to rehearse the stimuli silently and to remember them serially in order to repeat the sequence aloud after presentation of each list (the end of the list being indicated by a question mark). The response time allowed was 5,000 millisec (Figure la). In the updating working-memory task (adapted from Morris and Jones, 1990; see also Van der Linden et al., 1994), lists of 4, 6, 8 , and 10 consonants were presented at a rate of one every two seconds. Subjects were not informed of the length of each list before presentation. They were asked to rehearse silently and to remember serially only the last four items. They had to repeat those four items aloud after the presentation of each list (the end of the list being indicated by a question mark). The response time allowed was 5,000 millisec (Figure lb).

Sequences that sounded like words or abbreviations were avoided. For the updating task, the various lists were presented in a randomised order, with the restriction that no more than two lists of the same length were presented in succession. Subjects' responses were recorded on tape. The control and experimental tasks consisted of six sequences, with each task lasting $135 \mathrm{sec}$. In order to match the number of visual presentations between the two tasks, sharps (\#) were inserted between trials in the control task that subjects did not have to process. 


\section{Word Condition}

The second condition involved the storage and updating of semantic information. The material consisted of 36 French mono-, bi- and trisyllabic words. These words belonged to six different semantic categories (fishes, birds, vegetables, fruits, clothes and tools). The selected words were not prototypes of their semantic categories and the frequency of occurrence of the words was similar for all six semantic categories $[\mathrm{F}(5,30)=1.48, \mathrm{p}>.1]$. Words were presented one at a time on a visual display (80-point Arial Narrow font) while the names of the semantic categories used were displayed throughout the trial. Subjects responded aloud.

In the control working memory task, exemplars of different semantic categories were presented at a rate of one every two seconds and the participants memorised only the exemplars belonging to a specified semantic category (this category was displayed at the top of the screen during the whole trial). They had to freely recall those items aloud after presentation of each list (the end of the list being indicated by a question mark). The response time allowed was 5,000 millisec. The length of the sequence was unknown (from 7 to 11 words) and the number of exemplars to be memorised by trial was three or four (Figure 2a). In the updating working memory task, exemplars belonging to three or four different semantic categories were sequentially presented at a rate of one every two seconds while the names of the categories remained at the top of the screen. Participants had to remember only the last exemplar presented in each category in order to recall them freely at the end of the series (which was indicated by a question mark). The response time allowed was 5,000 millisec. The length of the sequence was unknown (from 7 to 11 words) and the number of exemplars to be memorised per trial was three or four (Figure $2 b)$.

For the control and experimental tasks, the lists of various lengths were presented in a randomised order, with the restriction that no more than two lists of the same length were presented successively. Subject responses were recorded on tape. The control and experimental tasks consisted of four sequences, with each task lasting $153 \mathrm{sec}$. In order to match the number of visual presentations between the two tasks, sharps (\#) were inserted between trials in the control task that subjects did not have to process.

Fig. 3 - Schematic illustration of cognitive components involved in sounds updating: (a) control task, (b) experimental updating task.

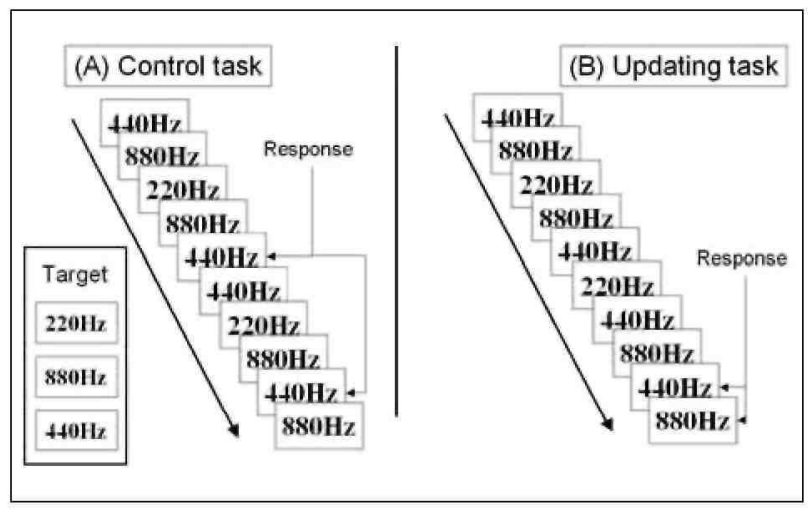

\section{Sound Condition}

Three kinds of sounds were created using the Praat 3.8.6.1 software (Boersma and Weenink, 2003): low-pitch $(880 \mathrm{~Hz})$, middle-pitch $(440 \mathrm{~Hz})$ and high-pitch $(220 \mathrm{~Hz})$ tones. These sounds were presented to subjects through earphones. The task was composed of three trials, with each trial corresponding to the random presentation of 21 sounds. Each sound was presented for 500 millisec, with an interstimulus interval of 1,500 millisec.

In the control working memory task, subjects had to press a response key to signal each occurrence of a predetermined sequence of three sounds (e.g., $440 \mathrm{~Hz}, 220 \mathrm{~Hz}, 880 \mathrm{~Hz}$ ), which corresponded to a memory load of three items. A target sequence was presented before the onset of each trial (Figure 3a). The updating working memory task consisted of detecting the fourth occurrence of each kind of tone. This required subjects to update, throughout the task, the number of times the low-, medium- and high-pitched tones had been presented (Figure $3 b)$. 
The experimental task lasted $146 \mathrm{sec}$ and the number of correct detections of the fourth occurrence of each kind of tone was recorded. With regard to the control task, the duration was $165 \mathrm{sec}$ (due to the supplementary presentation of the target sequence) and the number of correct key-presses following the presentation of the target sequence was recorded.

Subjects were trained five or six days before the PET session. Three minutes before each acquisition, the instructions were rehearsed. After the scanning session, post hoc questioning of the subjects indicated that they had perfectly complied with the task instructions. Each of the six tasks was performed twice during the session; tasks were counterbalanced between subjects to control for order effects.

PET data were acquired on a Siemens CTI 951 R 16/31 scanner in 3D mode. The subject's head was stabilised by a thermoplastic facemask secured to the head holder (TruScan Imaging, Annapolis, MD, USA), and a venous catheter was inserted in a left antebrachial vein. First, a 20-minute transmission scan was acquired for attenuation correction using three rotating sources of $68 \mathrm{Ge}$. Then, regional cerebral blood flow, taken as a marker of local neuronal activity (Jueptner and Weiller, 1998), was estimated during 12 emission scans. Each scan consisted of two frames: a 30-sec background frame and a 90-sec acquisition frame. The slow intravenous water $\left(\mathrm{H}_{2}{ }^{15} \mathrm{O}\right)$ infusion began $10 \mathrm{sec}$ before the second frame. Six mCi (222 MBq) were injected for each scan, in $5 \mathrm{cc}$ saline, over a period of $20 \mathrm{sec}$. The infusion was totally automated in order to avoid disturbing the subject during the scanning period. Data were reconstructed using a Hanning filter (cut-off frequency: 0.5 cycles/pixel) and corrected for attenuation and background activity. The cognitive tasks were randomly distributed between subjects, with the exception that no cognitive task was administered twice in succession and that no more than two experimental or control tasks were administered in succession.

\section{Data Analysis}

The PET data were analysed using Statistical Parametric Mapping (SPM99; Wellcome Department of Cognitive Neurology, Institute of Neurology, London, UK: http://www.fil.ion.ucl.ac.uk/spm) implemented in MATLAB (MathWorks Inc, Natick, MA, USA). For each subject, all scans were realigned together, then normalised to a standard PET template using the same transformations (Frackowiak et al., 1997). Finally, PET images were smoothed using a Gaussian kernel of $16 \mathrm{~mm}$ full width at half maximum to accommodate intersubject differences in gyral and functional anatomy and to suppress high-frequency noise in the images. Such transformations of the data allow for voxel-by-voxel averaging of data across subjects and for direct crossreference to the anatomical features in the standard stereotactic atlas (Talairach and Tournoux, 1988).

Differences in global activity within and between subjects were removed by analysis of covariance on a voxelby-voxel basis with global count as covariate and regional activity across subjects for each task as treatment (Friston et al., 1990). The condition and subject (block) effects were estimated according to the general linear model at each voxel, using a random effect model. In SPM99, the random effect model is a two-step procedure applied to accommodate intra-individual and interindividual variability of PET data, thus explicitly accounting for subject-by-condition interaction effects. In the first step, fixed-effects analyses were performed at the withinsubject level using linear contrasts of condition estimates. For each individual, subtraction contrasts were computed separately ( $\mathrm{p}<.001$, uncorrected). The resulting estimates (i.e., individual contrast images) fitted the within-subject component of variance. In the second set of analyses, the residual between-subject variance was assessed comparing individual estimates created at the first level. The resulting set of voxel values for each contrast constituted a map of the $t$ statistic $[\operatorname{SPM}(\mathrm{t})]$, thresholded at $\mathrm{p}<.001(\mathrm{~T}<2.61)$. Statistical inferences were then obtained at the voxel level (in terms of peak height at $\mathrm{p}<.05$ ), corrected for multiple comparisons. SPM was thresholded to $\mathrm{p}<.001$ (uncorrected for multiple comparisons) in the subtraction and functional connectivity analysis, when looking for activation in a region predicted by the conjunction analysis. In order to determine which cerebral areas were common to the three updating tasks, a conjunction analysis was performed in which the changes in cerebral activity common to the comparison of the updating and control tasks (for consonants, words and sounds) were assessed. Moreover, subtraction analysis between the updating and control tasks was also performed for the three conditions separately. This was done to determine the cerebral areas specific to the updating process for consonants, words and sounds.

Finally, the relationships between the cerebral areas commonly involved in the updating process were assessed using functional connectivity in a fixed-effect analysis. The design matrix included the same scans as described above (namely, the three updating conditions and the three control conditions in our twelve subjects). Now the analysis looked for brain regions that experienced a significant difference in reciprocal modulation with/from the cortical regions associated to the three updating tasks. It included the most significant peaks of the 10 different Brodmann areas (voxel level corrected $\mathrm{p}<.05$ ) obtained from the conjunction analysis and assessed the 
difference in modulation of every of the 10 voxels depending on the condition (updating or control). The results were considered significant at the voxel level, $\mathrm{p}<.001$, uncorrected for multiple comparisons when corresponding to cerebral areas evidenced by the conjunction analysis.

\section{RESULTS}

\section{Neuropsychological Performance}

The number of correct responses for the control and experimental tasks in each condition (in percent, mean \pm standard deviation) was analysed using a Friedman non-parametric analyses of variance with repeated measures (see Table I). The results showed that the response accuracies for the three updating tasks were not different [F $(2,11)=2.67, \mathrm{p}>.1]$ and that performance was better on the control tasks than the updating tasks [consonants: $\mathrm{F}$ $(1,11)=47.1, \mathrm{p}<.0001$; words: $\mathrm{F}(1,11)=163.8, \mathrm{p}<.0001$; sounds: $\mathrm{F}(1,11)=26.9, \mathrm{p}<.005]^{1}$.

TABLE I - Neuropsychological performance

\begin{tabular}{llll}
\hline \multicolumn{3}{l}{ Subjects' cognitive performance in the three conditions } \\
\hline & Consonants & Words & Sounds \\
\hline Updating task & $79 \pm 18$ & $76.3 \pm 11$ & $66.3 \pm 23$ \\
Control task & $98.7 \pm 3$ & $99.6 \pm 2$ & $87.4 \pm 17$ \\
\hline
\end{tabular}

Note. Percentage of correct responses (mean \pm standard deviation) in the three conditions.

\section{Imaging Data}

\section{Conjunction Analysis}

In order to determine which cerebral areas were involved in the three updating tasks, a conjunction analysis was performed in which changes in cerebral activity common to the comparison of updating tasks to control storage tasks (for consonants, words and sounds together) were assessed. This analysis showed increases in activity ( $\mathrm{p}<$ .05 , corrected) in the left frontopolar cortex (BA 10), in the left (BA 9) and right middle frontal gyrus (BA 9/46) and bilaterally in the superior frontal sulcus (BA 6). Foci of cerebral activation were also found in the left inferior frontal (BA 44) and right lateral orbitofrontal (BA 11) areas. Increased cerebral activity was also found bilaterally in the intraparietal sulcus (BA 7) and in the right inferior parietal gyrus (BA 40). Finally, foci of increased cerebral activity were found in the medial and right cerebellum (Table II and Figure 4).

\section{Subtraction Analysis}

The comparison of the updating and storage tasks in the consonant condition demonstrated increased cerebral activity in the left frontopolar cortex (BA 10) ( $p<.05$, corrected). Additional foci of activation ( $<<.001$, uncorrected) were also found in several areas already identified by the conjunction analysis: the right middle frontal gyrus (BA 9/46), the left superior frontal sulcus (BA 6), the right inferior parietal region (BA 40) and the medial cerebellum (Table III).

With regard to the word condition, there were significant foci of activation ( $\mathrm{p}<.001$, uncorrected) in a series of regions displayed in the conjunction analysis: the left frontopolar cortex (BA 10), the middle frontal gyrus bilaterally (BA 9/46), the left superior frontal sulcus (BA 6), as well as the right intraparietal sulcus (BA 7), the right inferior (BA 40) parietal gyrus, and the cerebellum bilaterally (Table IV).

Finally, when the sound updating working memory task was compared to the control storage task, an increase in cerebral activity was found in the left middle frontal gyrus (BA 9) ( $p<.05$, corrected). Supplementary foci of activation ( $\mathrm{p}<.001$, uncorrected) also existed in the right middle frontal area (BA 46), the right inferior parietal area (BA 40) and left intraparietal sulcus (BA 7), and finally the medial and right cerebellum (Table V).

\footnotetext{
${ }^{1}$ In order to assess the influence of task difficulty on cerebral activity, the different conditions were also compared, with individual performance as the confounding covariate. This analysis yielded similar results to those reported in Tables II-IV.
} 
TABLE II - Regions with significant regional cerebral blood flow (rCBF) changes in the comparison of the three updating tasks to the three control tasks (conjunction analysis)

\begin{tabular}{|c|c|c|c|c|}
\hline \multirow[t]{2}{*}{ Cerebral areas } & \multicolumn{3}{|c|}{ Stereotactic co-ordinates } & \multirow[t]{2}{*}{ Z score } \\
\hline & $\bar{X}$ & $y$ & $z$ & \\
\hline \multicolumn{5}{|c|}{ Voxel p-value $<.05$, corrected for multiple comparisons } \\
\hline \multirow[t]{3}{*}{ L frontopolar cortex (BA 10) } & -30 & 49 & 1 & 7.80 \\
\hline & -32 & 49 & 10 & 6.69 \\
\hline & -34 & 39 & 11 & 5.45 \\
\hline \multirow[t]{2}{*}{ L inferior frontal sulcus (BA 9) } & -40 & 22 & 21 & 5.51 \\
\hline & -53 & 17 & 27 & 5.94 \\
\hline \multirow[t]{2}{*}{ R middle frontal gyrus (BA 9/46) } & 48 & 42 & 20 & 5.51 \\
\hline & 48 & 34 & 22 & 5.60 \\
\hline \multirow[t]{2}{*}{ L and R superior frontal sulcus (BA 6) } & -26 & 7 & 53 & 5.28 \\
\hline & 32 & -1 & 50 & 5.26 \\
\hline L inferior frontal gyrus (BA 44/45) & -61 & 17 & 19 & 5.06 \\
\hline $\mathrm{R}$ lateral orbitofrontal cortex (B A 11/10) & 42 & 56 & -16 & 5.25 \\
\hline \multirow[t]{2}{*}{ L and $\mathrm{R}$ intraparietal sulcus (BA 7) } & -26 & -58 & 47 & 5.82 \\
\hline & 48 & -59 & 55 & 5.89 \\
\hline R inferior parietal gyrus (BA 40) & 55 & -37 & 42 & 6.68 \\
\hline \multirow[t]{3}{*}{ Medial cerebellum } & 2 & -71 & -30 & 5.86 \\
\hline & 2 & -61 & -21 & 5.98 \\
\hline & 4 & -63 & -12 & 6.02 \\
\hline \multirow[t]{2}{*}{$\mathrm{R}$ cerebellum } & 51 & -50 & -27 & 5.31 \\
\hline & 18 & -67 & -19 & 5.36 \\
\hline
\end{tabular}

Note. Coordinates and Z-scores for voxels in which there were significant activation foci when the updating tasks were compared to the control tasks. $\mathrm{L}=$ left hemisphere; $\mathrm{R}=$ right hemisphere. $x, y, z$ (in $\mathrm{mm}$ ) refer to coordinates in the Talairach space (Talairach and Tournoux, 1988).

TABLE III - Regions with significant regional cerebral blood flow ( $r C B F)$ changes in the comparison of the consonant running span task to the matched control task (subtraction analysis)

\begin{tabular}{|c|c|c|c|c|}
\hline \multirow[t]{2}{*}{ Brain areas } & \multicolumn{3}{|c|}{ Stereotactic co-ordinates } & \multirow[t]{2}{*}{ Z score } \\
\hline & $x$ & $y$ & $z$ & \\
\hline \multicolumn{5}{|c|}{ Voxel p-value $<.05$, corrected for multiple comparisons } \\
\hline L frontopolar cortex (BA 10) & -30 & 49 & 1 & 5.44 \\
\hline \multicolumn{5}{|c|}{ Voxel p-value $<.001$, uncorrected for multiple comparisons } \\
\hline R middle frontal gyrus (BA 9/46) & 46 & 46 & 18 & 3.43 \\
\hline & 44 & 40 & 27 & 3.94 \\
\hline L superior frontal sulcus (BA 6) & -30 & 3 & 53 & 3.75 \\
\hline L inferior frontal gyrus (BA 44/45) & -61 & 18 & 18 & 4.03 \\
\hline $\mathrm{R}$ inferior parietal gyrus (BA 40) & 55 & -37 & 46 & 4.77 \\
\hline Medial cerebellum & 2 & -61 & -7 & 3.58 \\
\hline
\end{tabular}

Note. Coordinates and Z-scores for voxels in which there were significant activation foci when the consonant updating task was compared to the matched control task. $\mathrm{L}=$ left hemisphere; $\mathrm{R}=$ right hemisphere. $x, y, z$ (in $\mathrm{mm}$ ) refer to coordinates in the Talairach space (Talairach and Tournoux, 1988). 
Fig. 4 - Brain activation observed in the conjunction analysis between the three updating tasks (in comparison to their respective control tasks). Co-ordinates of all significant regions are given in Table II. Brain areas are rendered on a standard brain conforming to stereotactic space in SPM99 (corrected p value $<.05$ ).

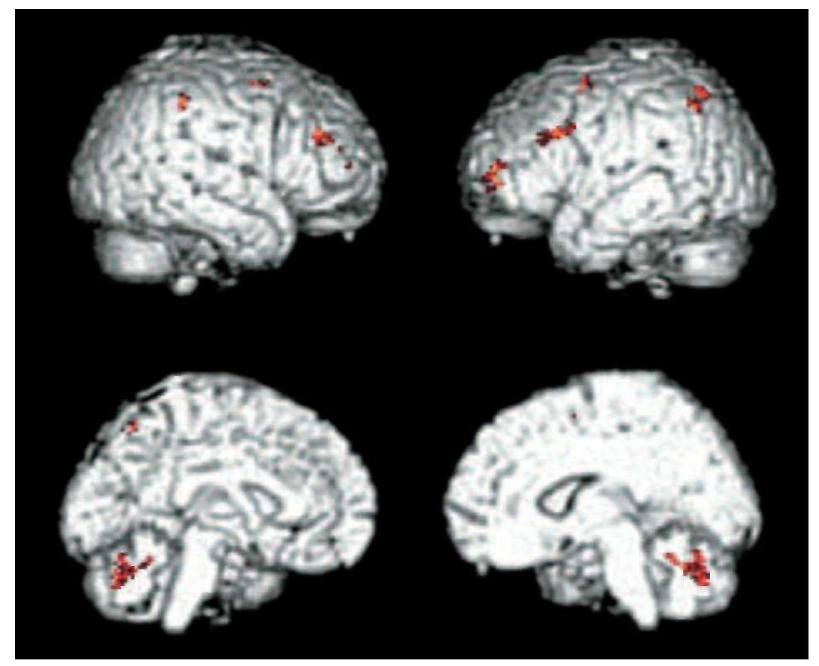

TABLE IV - Regions with significant regional cerebral blood flow ( $r C B F)$ changes in the comparison of the word running span task to the matched control task (subtraction analysis)

\begin{tabular}{|c|c|c|c|c|}
\hline \multirow[t]{2}{*}{ Brain areas } & \multicolumn{3}{|c|}{ Stereotactic co-ordinates } & \multirow[t]{2}{*}{ Z score } \\
\hline & $x$ & $y$ & $z$ & \\
\hline \multicolumn{5}{|c|}{ Voxel p-value $<.001$, uncorrected for multiple comparisons } \\
\hline L frontopolar cortex (BA 10) & -30 & 49 & 1 & 4.66 \\
\hline \multirow[t]{3}{*}{$\mathrm{L}$ and $\mathrm{R}$ middle frontal gyrus (BA 9/46) } & -50 & 21 & 27 & 3.98 \\
\hline & -34 & 44 & 18 & 3.36 \\
\hline & 50 & 36 & 18 & 3.22 \\
\hline L superior frontal sulcus (BA 6) & -24 & 12 & 51 & 3.35 \\
\hline $\mathrm{R}$ intraparietal sulcus (BA 7) & 44 & -59 & 56 & 4.14 \\
\hline $\mathrm{R}$ inferior parietal gyrus (BA 40) & 55 & -37 & 41 & 3.35 \\
\hline \multirow[t]{2}{*}{$\mathrm{L}$ and $\mathrm{R}$ cerebellum } & -42 & -61 & -19 & 3.62 \\
\hline & 12 & -71 & 24 & 4.71 \\
\hline
\end{tabular}

Note. Coordinates and Z-scores for voxels in which there were significant activation foci when the word updating task was compared to the matched control task. $\mathrm{L}=$ left hemisphere; $\mathrm{R}=$ right hemisphere. $x, y, z$ (in $\mathrm{mm}$ ) refer to coordinates in the Talairach space (Talairach and Tournoux, 1988)

TABLE $\boldsymbol{V}$ - Regions with significant regional cerebral blood flow $(r C B F)$ changes in the comparison of the sound running span task to the control task (subtraction analysis)

\begin{tabular}{|c|c|c|c|c|}
\hline \multirow[t]{2}{*}{ Cerebral areas } & \multicolumn{3}{|c|}{ Stereotactic co-ordinates } & \multirow[t]{2}{*}{ Z score } \\
\hline & $x$ & $y$ & $z$ & \\
\hline \multicolumn{5}{|c|}{ Voxel p-value $<.05$, corrected for multiple comparisons } \\
\hline L middle frontal gyrus (BA 9) & -46 & 23 & 32 & 4.96 \\
\hline \multicolumn{5}{|c|}{ Voxel p-value $<.001$, uncorrected for multiple comparisons } \\
\hline R middle frontal gyrus (BA 46) & 46 & 46 & 18 & 4.48 \\
\hline $\mathrm{R}$ inferior parietal gyrus (BA 40) & 50 & -60 & 53 & 4.19 \\
\hline L intraparietal sulcus (BA 7) & -28 & -60 & 51 & 3.71 \\
\hline Medial cerebellum & 8 & -75 & -23 & 4.46 \\
\hline $\mathrm{R}$ cerebellum & 59 & -50 & 26 & 3.80 \\
\hline
\end{tabular}

Note. Coordinates and Z-scores for voxels in which there were significant activation foci when the sound updating task was compared to the matched control task. $\mathrm{L}=$ left hemisphere; $\mathrm{R}=$ right hemisphere. $x, y, z$ (in $\mathrm{mm}$ ) refer to coordinates in the Talairach space (Talairach and Tournoux, 1988) 
Functional connectivity (Friston and Price, 2001) between the cerebral areas commonly associated with the three updating tasks and the whole brain metabolism was also assessed (see Table VI and Figure 5). This analysis demonstrated correlations between remote cerebral areas that existed in the three updating tasks but not in the three control tasks. First, functional connectivity of frontal areas was assessed. The left frontopolar cortex (BA 10) showed a preferential connectivity in updating tasks over storage tasks with the right inferior frontal gyrus (B A 44) and right inferior parietal area (BA 40). The left middle frontal gyrus (BA 9) was functionally connected to the right middle frontal gyrus (BA 9/46), as well as the left superior frontal sulcus (BA 6) and the right inferior frontal gyrus (BA 45). The right middle frontal gyrus (BA 9/46) was connected to the left frontopolar (BA 10) and middle frontal gyrus (BA 9), bilaterally to another area of the middle frontal gyrus (BA 6), and to the left intraparietal sulcus (BA 7) and right inferior parietal (BA 40) gyrus, as well as to the medial cerebellum. Finally, the left inferior frontal gyrus (BA 44) showed connectivity with the right frontopolar cortex (BA 10). With regard to parietal areas, the left intraparietal sulcus (BA 7) showed functional connectivity with two lefthemisphere areas: the lateral orbitofrontal gyrus (BA 10) and the superior frontal sulcus (BA 6). The right intraparietal sulcus (BA 7) was also correlated with two left-hemisphere areas - the frontopolar cortex (BA 10) and the middle frontal gyrus (BA 46) - as well as with the right cerebellum. Finally, the right inferior parietal area (BA40) showed functional correlations with the right inferior frontal (BA 44) and the right intraparietal sulcus (BA 7). The medial cerebellum was functionally linked to the left and right inferior frontal gyrus (respectively, BA 44 and 45). Functional connectivity was demonstrated between the right cerebellum and bilateral middle frontal areas (left BA 46 and right BA 6).

Fig. 5 - Schematic illustration of the functional connectivity between cerebral areas found in the conjunction analysis and the whole brain metabolism.

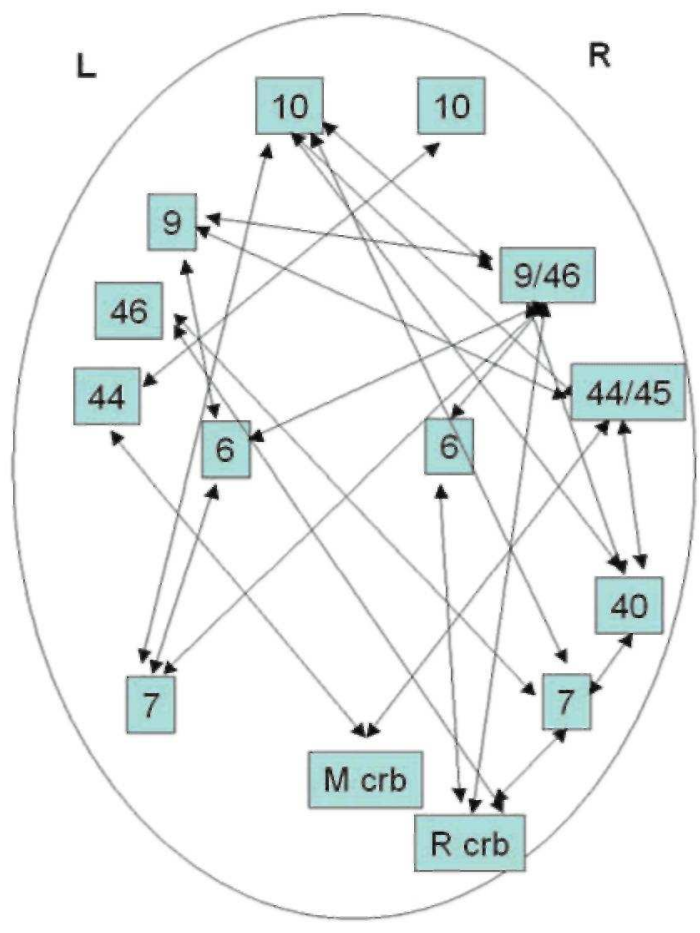


TABLE VI - Regions demonstrating functional connectivity with cerebral areas evidenced in the conjunction analysis

\begin{tabular}{llll}
\hline Cerebral areas & \multicolumn{2}{l}{ Stereotactic co-ordinates } & Z score \\
\cline { 2 - 3 } & $x$ & $y$
\end{tabular}

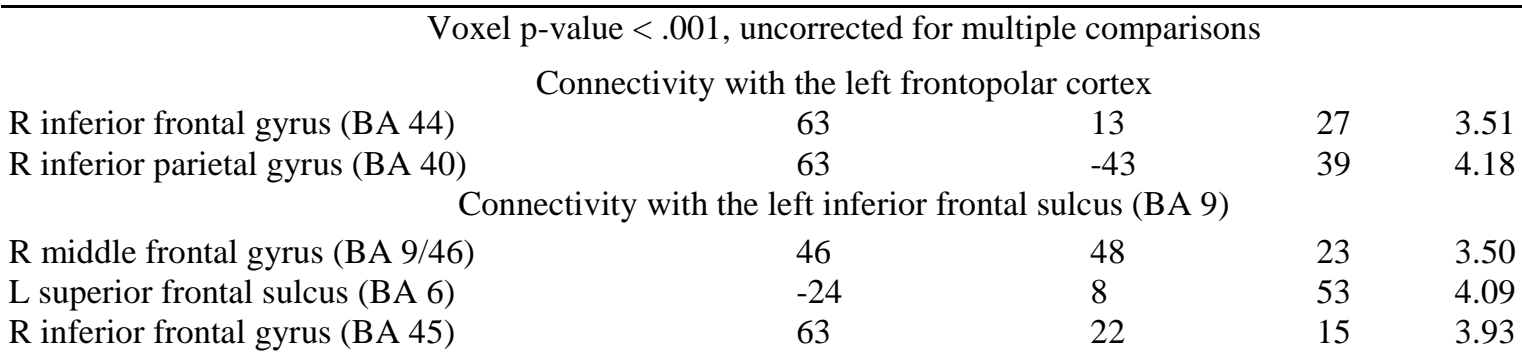

$\mathrm{R}$ inferior frontal gyrus (BA 45)

Connectivity with the right middle frontal gyrus (BA 9/46)

$\begin{array}{lllll}\text { L frontopolar cortex (BA 10) } & -26 & 58 & 4 & 3.21 \\ \text { L middle frontal gyrus (BA 9) } & -46 & 23 & 27 & 3.45 \\ \text { L and R middle frontal gyrus (BA 6) } & -38 & 8 & 49 & 3.45 \\ & 26 & -3 & 54 & 3.33 \\ \text { L intraparietal sulcus (BA 7) } & -40 & -61 & 56 & 3.39 \\ \text { R inferior parietal gyrus (BA 40) } & 50 & -54 & 56 & 3.56 \\ \text { R cerebellum } & 18 & -69 & -20 & 3.63\end{array}$

Connectivity with the left inferior frontal gyrus (BA 44)

$\begin{array}{lcccr}\text { R frontopolar cortex (BA 10) } & 30 & 55 & 5 & 3.14 \\ & \text { Connectivity with the left intraparietal sulcus } & & \\ \text { L lateral orbitofrontal gyrus (BA 10) } & -42 & 46 & -10 & 3.53 \\ \text { L superior frontal sulcus (BA 6) } & -30 & 5 & 59 & 3.25\end{array}$

Connectivity with the right intraparietal sulcus

L frontopolar cortex (BA 10)

L middle frontal gyrus (BA 46)

$\mathrm{R}$ cerebellum

$\begin{array}{llll}-30 & 51 & 1 & 3.16 \\ -46 & 32 & 17 & 3.17 \\ 53 & -69 & -23 & 3.53 \\ 20 & -61 & -15 & 3.33\end{array}$

Connectivity with the right supramarginal gyrus (BA 40)

$\mathrm{R}$ inferior frontal gyrus (BA 44)

$R$ intraparietal sulcus (BA 7)

L inferior frontal gyrus (BA 44)

$\mathrm{R}$ inferior frontal gyrus (BA 45)

L middle frontal gyrus (BA 46)

$\mathrm{R}$ middle frontal gyrus (BA 6)
53

38

16

$-52$

Connectivity with the medial cerebellum

$-59 \quad 17$

$65 \quad 20$

20

Connectivity with the right cerebellum

$\begin{array}{llll}-48 & 41 & 9 & 3.83 \\ 30 & -3 & 48 & 3.25\end{array}$

Note. Coordinates and Z-scores for voxels in which there were significant activation foci when functional connectivity with areas found in the conjunction analysis was assessed. $\mathrm{L}=$ left hemisphere; $\mathrm{R}=$ right hemisphere. $x, y, z$ (in $\mathrm{mm}$ ) refer to coordinates in the Talairach space (Talairach and Tournoux, 1988).

\section{DISCUSSION}

The results obtained in this study can be summarised as follows. First, our conjunction and subtractions analyses demonstrated that there is a network of cerebral areas common to the three updating tasks, although some areas appear more specific to each task. More specifically, a bilateral network of prefrontal, parietal and cerebellar regions subserved performance on all three updating tasks while, within this network, some areas appeared more engaged by one updating task or another; the left frontopolar (BA 10) for consonant updating and the left middle frontal gyrus (BA 9) for sound updating and, at a lower statistical threshold, the left frontopolar and right cerebellum for word updating. Finally, functional connectivity analyses demonstrated a large number of interrelationships between the different cerebral areas involved in the updating function. 
Since the cerebral network common to the three updating tasks was evidenced with a conjunction analysis, these areas should be relatively independent of the exact characteristics of the tasks to be performed, except the requirement to update the presented information during the task. However, the large number of cerebral areas associated with the updating function leads one to question the unitary nature of this process. In fact, as suggested by Miyake et al. (2000), the identification of target executive functions such as updating, shifting, or inhibition constitutes a useful level of analysis but these functions can also be decomposed into more basic component processes. In particular, besides encoding and maintenance processes, the updating function depends on a series of processes such as shifting from internal to external information, discarding irrelevant items, tracking serial order, and repositioning items.

Among the different areas demonstrating an activation common to all three updating tasks, the frontopolar cortex has previously been found to be associated with performance on the running span task (Salmon et al., 1996; Van der Linden et al., 1999a). It has been proposed that this region supports the processing of internally generated or stimulus-independent thought, and thus that it is active in situations involving the evaluation and selection of internally generated information (Christoff and Gabrieli, 2000; Christoff et al., 2001, 2003; McGuire et al., 1996). Another account of frontopolar function is that this region is involved in a so-called 'branching' cognitive process, consisting of carrying out a secondary task while holding a primary task in working memory (Koechlin et al., 1999). Both of these functions could actually be combined in a recent proposal by Burgess et al. (2005), which suggests that the frontopolar region plays a role in co-ordinating attention between internally represented and externally presented information, without being directly responsible for information transformations (the 'gateway hypothesis'). The updating memory tasks we used required subjects to maintain an internal representation in the absence of external cues (the number of items already presented), while simultaneously processing externally presented stimuli (the continuous presentation of new items).

Many functions have been attributed to the middle/inferior lateral prefrontal cortex: manipulation of information (Collette et al., 1999; D'Esposito et al., 1999; Postle et al., 1999), dual task co-ordination (D'Esposito et al., 1995), shifting processes (Rogers et al., 2000), and inhibition (Chee et al., 2000; Collette et al., 2001). Owen et al. (2000) proposed that a general role for the ventrolateral frontal cortex in working memory would be to trigger active low-level encoding strategies (such as those involved in the forward span task, requiring a relatively straightforward mapping of stimulus to response). In contrast, the dorsolateral frontal cortex would be activated in memory situations that require subjects to monitor responses made and information assimilated earlier in the trial (such as free recall or backward digit span). However, Raye et al. (2002) proposed that activity in the dorsolateral prefrontal cortex (BA 9) is recruited to prolong (or increase) the activation of representations, or resuscitate those with sufficient levels of activity (a 'refreshing' process). Both these interpretations (monitoring and refreshing) appear valuable in the context of updating and further studies will be necessary to determine the exact function of the dorsolateral prefrontal cortex during running span tasks.

We also observed increased cerebral activity in parietal areas during updating. Such activity had already been described in previous studies (e.g., Braver et al., 1997) and was most frequently explained in terms of storage function (e.g., Honey et al., 2000). However, increased cerebral activity was found in the left posterior parietal region (more specifically, in the intraparietal sulcus), close to the area described by Marshuetz et al. (2000) as underlying ordination processes. Tracking of serial order and repositioning items in working memory are considered to be two major updating processes (Postle et al., 2001). Other regions associated with ordination processes are the superior frontal areas and cerebellum (Henson et al., 2000). It must, however, be noted that bilateral activity in the intraparietal sulcus, found in a study by LaBar et al. (1999), was common to a verbal working memory task and a spatial attention task. The authors proposed that this neuroanatomic overlap relates to the shifting of an attentional focus, irrespective of whether the shifts occur over space, time or cognitive domain. Finally, we have no clear interpretation of the role of the right lateral orbitofrontal cortex (BA 11/10) at present, but this region has been associated with inhibitory processes (for a review, see Collette and Van der Linden, 2002).

Functional connectivity analyses were also performed to determine the cerebral areas whose activity covaried with regions evidenced by the conjunction analyses. Some of these interrelationships are consistent with our hypotheses concerning the functional role of these regions. In particular, they fit the interpretation suggesting that the frontopolar cortex acts as a gateway between the processing of internal representations and externally presented stimuli, directing the flow of information between regions where transformations are realised (Burgess et al., 2005). Indeed, we found that activity in the left frontopolar cortex was associated with the right middle and inferior frontal gyrus. According to Owen (2000), the inferior frontal gyrus may be associated with the processing of externally presented information (since this area is involved in low-level encoding strategies) and the middle frontal gyrus with the processing of internal representations (i.e., monitoring of information 
presented earlier). In that context, the role of the frontopolar cortex would be to hedge the rote rehearsal of information (a low-level encoding strategy devoted to the inferior frontal gyrus) and to reorganise this information following the presentation of newer items (a monitoring function devoted to the middle frontal gyrus). Similarly, we observed functional connectivity between the intraparietal sulcus, the cerebellum and the superior frontal sulcus. These areas have been claimed to be responsible for serial order processing (Marshuetz et al., 2000; Henson et al., 2000), and more specifically, the continuous repositioning of items in working memory (Postle et al., 2001). Interestingly, these regions were also recruited for updating of visuospatial information (Cornette et al., 2001). Finally, we also observed functional connectivity between the prefrontal and parietal areas. Although further studies are necessary to confirm this hypothesis, one might propose that, during updating, the prefrontal areas send a signal to the parietal regions (typically regarded as a 'storage' area ; e.g., Honey et al., 2000) in order to access and edit the current memory representation Due to the PET methodology (which generates a small number of functional imaging data per subject and condition), these functional connectivity analyses were performed on all three updating tasks simultaneously, and connectivity specific to each updating task was not assessed. Moreover, these analyses showed cerebral areas where activity covaries but did not allow us to determine which of the areas influenced the functional response of the other. In that context, it would be particularly fruitful to pursue these investigations using fMRI, which would allow one to contrast the functional connectivity specific to each task, and also to explore the modulation of the network evidenced here with regard to the subject's performance or, in other words, to determine whether some cerebral areas modulate the functional responses of others depending on the production of correct or erroneous responses.

Finally, it must be emphasised that the comparison of each updating task to the matched control task showed the most significant activity in various areas of this general common network. These results are in accordance with the view that, within this general network, some areas are more involved in one task than in another. The reasons for the differences in cerebral activity between tasks considered as mainly reflecting an updating ability are not really clear, and we cannot totally reject the hypothesis that some of these results are undermined by differences across conditions in task demands (e.g., memory load, differences in task parameters, etc.) although control tasks were cautiously selected to remove such influences. However, the following interpretations may be tentatively proposed. The region most associated with consonant updating is the left frontopolar cortex, considered as a gateway between the processing of internal representations and externally presented stimuli (Burgess et al., 2005), a key process for updating. Thus, consonant updating would be primarily characterised by this process, and repositioning of items or inhibition would be less involved in this task. Otherwise, although Miyake et al. (2000) demonstrated that the word running span task is clearly associated with an updating factor, no specific cerebral area was found to be particularly associated with this task at a corrected statistical threshold. However, some processes related to updating already exist in the control task. Indeed, this task requires participants to maintain in memory only exemplars belonging to a specified semantic category. This means they must inhibit the encoding of exemplars from other semantic categories and compare the presented information to internal semantic representations, two processes that are clearly associated with updating. Thus, the use of such a control task could have masked cerebral activity specific to semantic updating. It should also be noted that this task, considered as requiring updating, is also characterised by task-switching, because participants must keep track of four category representations at once, and switch attention between them. Finally, sound updating was mainly associated with the left middle frontal gyrus (BA 9). In comparison to the control task, sound updating requires subjects to keep track of the number of times a specific sound is presented. This induces a supplementary memory load, counting processes and manipulation of information without any need to reorganise it (contrary to the two previous updating tasks). In support of this hypothesis, the left middle frontal gyrus had previously been found to be associated with the manipulation of information in working memory (Collette et al., 1999) or refreshing just-presented information (Raye et al., 2002).

In conclusion, the results obtained with a conjunction analysis clearly demonstrate that there is a cerebral network associated with the updating function, whatever task is administered. Moreover, the existence of connections between cerebral areas within this network allowed to confirm the functional role attributed to these regions. So connections between the left frontopolar cortex and middle and inferior frontal gyrus are in agreement with the "gateway" function attributed to this region by Burgess et al. (2005). Networks associated with serial order processing and, more tentatively, with the editing of memory representations were also isolated. Finally, the results of subtraction analyses showed that the degree of activation of these areas depends on the specific characteristics of the updating task (i.e., the extent to which the task requires specific processes such as co-ordinating attention between internally represented and externally presented information, processing serial order, inhibiting irrelevant information, manipulating information, etc.) but also on the cognitive processes elicited by the matched control task. More generally, results obtained this study provide some information about the nature of executive functioning. In a previous study, Collette et al. (2005) demonstrated that these processes are characterised by unity and diversity at a neuronal level [see Miyake et al. (2000) for similar data at a 
cognitive level], since some cerebral areas are common to the executive processes of updating, shifting and inhibition while other areas appear specific to each process. With regard to the updating function, we observed a common network for the three tasks, but the use of the cerebral areas making up this network seems to be modulated depending on the exact characteristics of the tasks. These data could indicate that the updating process is characterised more by unity than by diversity of processes. However, this pattern was not observed for all executive functions: tasks identified by Miyake et al. (2000) as loading on a shifting factor require distinct cerebral areas, rather than a few common regions (Salmon et al., 2006); this finding is consistent with the conceptualisation of shifting as requiring mainly a diversity of process. Although further studies will be necessary to corroborate these hypotheses, it seems that the cognitive functions grouped together as "executive" can be characterized by both unity and diversity of functioning, and that the proportion of these two mode of functioning varies between executive processes.

\section{Acknowledgements}

Fabienne Collette and Steven Laureys are Research Associates at the National Fund for Scientific Research (FNRS) of Belgium. This study was supported by the Belgian FNRS, the Fondation Médicale Reine Elisabeth and an Interuniversity Attraction Pole (P5/04).

\section{References}

Awh E, Jonides J, Smith EE, Schumacher EH, KoEPPE RA and KATZ S. Dissociation of storage and rehearsal in verbal working memory: Evidence from positron emission tomography. Psychological Science, 7: 25-31, 1996.

Belleville S, RoulEAU N and CAZA N. Effects of normal aging on the manipulation of information in working memory. Memory and Cognition, 26: 572-583, 1998.

BJork RA. The updating of human memory. In Bower GH (Ed), The Psychology of Learning and Motivation. New York: Academic Press, 1978 .

BOERSMA P and WEENINK D. Praat-A system for doing phonetics by computer [computer software]. The Netherlands: Institute of Phonetic Sciences, University of Amsterdam, 2003.

Braver TS, CohEn JD, Nystrom LE, Jonides J, SMith EE and Noll DC. A parametric study of prefrontal cortex involvement in human working memory. Neurolmage, 5: 49-62, 1997.

BuRgESS PW, SimOns JS, DUMONTHEIL I and GILBERT SJ. The gateway hypothesis of rostral prefrontal cortex (area 10) function In Duncan J, McLeod P and Phillips L (Eds), Measuring the Mind: Speed, Control, and Age. Oxford: Oxford University Press, 2005.

Chee MW, Sriram N, Siong Soon C and Ming LeE K. Dorsolateral prefrontal cortex and the implicit association of concepts and attributes. Neuroreport, 11: 135-140, 2000. CHRISTOFF K and GABRIELLI JD. The frontopolar cortex and human cognition: Evidence for a rostrocaudal hierarchical
organization within the human prefrontal cortex. Psychobiology 28: 168-186, 2000.

Christoff K, Prabhakaran V, Dorfman J, Zhao Z, Kroger JK, HolyaK KJ and Gabrieli JD. Rostrolateral prefrontal cortex involvement in relational integration during reasoning. Neurolmage, 14: 1136-1149, 2001.

CHRISTOFF K, REAM JS, GEDDES LP and GABRIELI JD. Evaluating self-generated information: Anterior prefrontal contributions to human cognition. Behavioral Neuroscience, 117: 1161- 1168, 2003.

COHEN JD, Forman SD, Braver TS, CASEY BJ, SERVAN-SChreIBER D and Noll DC. Activation of the prefrontal cortex in a nonspatial working memory task with functional MRI. Human Brain Mapping, 1: 293-304, 1994.

COHEN JD, PERLSTEIN WM, BRAVER TS, NySTROM LE, Noll DC, JONIDES J and SMITH EE. Temporal dynamics of brain activation during a working memory task. Nature, 386: 604- 607, 1997.

Collette F, Salmon E, Van der Linden M, Chicherio C, Belleville S, Degueldre C, Delfiore G and Franck G Regional brain activity during tasks devoted to the central executive of working memory. Cognitive Brain Research, 7:411-417, 1999.

COLLETTE F and VAN DER LINDEN M. Brain imaging of the central executive component of working memory. Neuroscience and Biobehavioral Reviews, 26: 105-125,2002.

Collette F, VAn der Linden M, Delfiore G, Degueldre C, LuXen A and SAlmon E. The functional anatomy of inhibition processes investigated with the Hayling task. Neurolmage, 14: 258-267, 2001 
Published in : Cortex, (2007) 43, 146-158

Status : Postprint (Author's version)

Collette F, VAn Der Linden M, LAureys S, Del Fiore G, Degueldre C, LuXen A and Salmon E. Exploring the unity and diversity of the neural substrates of executive functioning. Human Brain Mapping, 25: 409-423, 2005.

CORnetTe L, Dupont P, SALMON E and ORBAn GA. The neural substrate of orientation working memory. Journal of Cognitive Neuroscience, 13: 813-828, 2001.

DE BENI R and PALladino P. Decline in working memory updating through ageing. Intrusion errors analyses. Memory, 12: 75-89. 2004.

D'Esposito M, Detre JA, Alsop CD, ShIn RK, ATLAS S and Grossman M. The neural basis of the central executive of working memory. Nature, 378: 279-281, 1995.

D'Esposito M, Postle BR, BALlard D and LeAse J. Maintenance versus manipulation of information held in working memory: An eventrelated fMRI study. Brain and Cognition, 41: 66-86. 1999.

FISK JE and SHARP CA. Age-related impairment in executive functioning: Updating, inhibition, shifting, and access. Journal of Clinical and Experimental Neuropsychology, 26: 874-890, 2004.

Frackowiak R, Friston K, Frith C, Dolan R and MazziotTa JC Human Brain Function. London: Academic Press, 1997.

FRISTON KJ, FRГГн CD, LIDDLE PF, DOLAN RJ, LAMмERTSMA AA and FRACKOWIAK RS. The relationship between global and local changes in PET scans. Journal of Cerebral Blood Flowand Metabolism, 10: 458-466, 1990.

FRISTON KJ and PRICE CJ. Generative models, brain functions and neuroimaging. Scandinavian Journal of Psychology, 42: 167- 177, 2001.

Friston KJ, Price CJ, Fletcher P, Moore C, Frackowiak RS and Dolan RJ. The trouble with cognitive substraction. Neurolmage, 4. 97-104, 1996.

GILBERT B, Belleville S, BHERER L and ChouinARD S. Study of verbal working memory in patients with Parkinson's disease. Neuropsychology, 19: 106-114, 2005.

HENSON RN, BURGESS N and FRITH CD. Recoding, storage, rehearsal and grouping in verbal short-term memory: An fMRI study. Neuropsychologia, 38: 426-440, 2000.

HONEY GD, Bullmore ET and Sharma T. Prolonged reaction time to a verbal working memory task predicts increased power of posterior parietal cortical activation. Neurolmage, 12: 495- 503, 2000.

Jonides J, Schumacher EH, Smith EE, LAUber EJ, Awh E, Minoshrma S and Koeppe RA. Verbal working memory load affects regional brain activation as measured by PET. Journalof Cognitive Neuroscience, 9: 462-475, 1997.

JUEPTNER M and WEILLER C. Review: Does measurement of regional cerebral blood flow reflect synaptic activity? Implications for PET and fMRI. Neurolmage 2: 148-156. 1998.

KOECHLIN E, BASSO G, PIETRINI P, PANZER S and GRAFMAN J. The role of the anterior prefrontal cortex in human cognition. Nature, 13: $148-51,1999$

LABAR KS, GITELMAN DR, PARRISH TB and MESUlAM MM. Neuroanatomic overlap of working memory and spatial attention nEtworks: A functional MRI comparison with subjects. Neurolmage, 10: 695-704, 1999.

MARShuEtz C, SMITH EE, Jonides J, DeGuTIS J and CHEVENERT TL. Order information in working memory: fMRI evidence for parietal and prefrontal mechanisms. Journal of Cognitive Neuroscience, 12: 130-144, 2000.

MCGUiRE PK, PAULeSU E, FraKOWIAK RS and FriTh CD. Brain activity during stimulus independent thought. Neuroreport, 7: 2095-2099, 1996.

MiYAKe A, Friedman NP, EMERSON MJ, WITZKi AH and HowERTER A. The unity and diversity of executive functions and their contribution to complex "frontal lobe" tasks: A latent variable analysis. Cognitive Psychology, 41: 49-100. 2000.

MORRIS N and JONES DM. Memory updating in working memory: The role of the central executive. British Journal of Psychology, 81: 111$121,1990$.

OWEN AM, LEE AC and WILLIAMS EJ. Dissociating aspects of verbal working memory within the human frontal lobes: Further evidence for a "process-specific" model of lateral frontal organisation. Psychobiology, 28: 146-155, 2000.

OWEn AM, Stern CE, LOOK RB, TRACEY I, Rosen BR and PETRIDES M. Functional organization of spatial and nonspatial working memory processing within the human lateral frontal cortex. Proceedings of the National Academy of Sciences of the USA, 95: 7721-7726, 1998. 
Published in : Cortex, (2007) 43, 146-158

Status : Postprint (Author's version)

POLLACK I, JOHNSON LB and KNAFF PR. Running memory span. Journal of Experimental Psychology, 57: 137-146, 1959.

POSTLE BR, BERGER JS and D'ESPOSITO M. Functional neuroanatomical double dissociation of mnemonic and executive control processes contributing to working memory performance. Proceedings of the National Academy of Sciences of the USA, 96: 12959-12964, 1999.

Postle BR, Berger J, Goldstein JH, CurTis CE and D'EsPosito M. Behavioral and neurophysiological correlates of episodic coding, proactive interference, and list length effects in a running span verbal working memory task. Cognitive, Affective, and Behavioral Neuroscience, 1: 10-21, 2001.

RAYE CL, Johnson MK, Mitchell KJ, REEDER JA and GREENE EJ. Neuroimaging a single thought: Dorsolateral PFC activity associated with refreshing just-activated information. Neurolmage, 15: 447-453, 2002.

ROGERS RD, ANDREWS TC, GRASBy PM, BROOKS DJ and ROBBINS TW. Contrasting cortical and subcortical activations produced by attentional-set shifting and reversal learning in humans. Journal of Cognitive Neuroscience, 12: 142-162,2000.

Salmon E, Van der Linden M, Collette F, Delfiore G, Maquet P, Degueldre C, Luxen A and Franck G. Regional brain activity during working memory tasks. Brain, 119: 1617-1625, 1996.

Salmon E, VAn der Linden M, VAndenberghe R, Delfiore G, Degueldre C, LuXen A and Collette F. Neural correlates of cognitive subcomponents in different shifting tasks. Submitted.

Schumacher EH, LAuber E, Awh E, Jonides J, Smith EE and KoePPE RA. PET evidence for an amodal verbal working memory system. Neurolmage, 3: 79-88, 1996

SHIMAMURA AP. The role of prefrontal cortex in dynamic filtering. Psychobiology, 28: 207-218, 2000.

SidTis JJ, STROTHER SC, ANDERSON JR and RotTENBERG DA. Are brain functions really additive? Neurolmage, 6: 490-496, 1999.

SMITH EE, JONIDES J and KoEPPE RA. Dissociating verbal and spatial working memory using PET. Cerebral Cortex, 6: 11-20, 1996.

TAlaIRACH J and Tournoux P. Co-planar Stereotaxic Atlas of the Human Brain: 3-Dimensional Proportional System: An Approach to Cerebral Imaging. Stuttgart: Thieme, 1988.

VAN DER LINDEN M, BRÉDART S and BEERTEN A. Age-related differences in updating working memory. British Journal of Psychology, 85. $145-152,1994$

VAn Der Linden M, Collette F, SAlmon E, Delfiore G, Degueldre C, LuXEN A and Franck G. The neural correlates of updating of information in verbal working memory. Memory, 7: 549-560, 1999a.

VAN DER Linden M, Hupet M, Feyereisen P, Schelstraete MA, Bestgen Y, Bruyer R, Lories G, EL AhMADi A and SERon X. Cognitive mediators of age-related differences in language comprehension and verbal memory performance. Aging, Neuropsychology and Cognition, 6: 32-55, 1999b. 